Article

\title{
Coefficient Estimates for a Subclass of Starlike Functions
}

\section{Dorina Răducanu $\mathbb{D}$}

Faculty of Mathematics and Computer Science, Transilvania University of Braşov, Iuliu Maniu,50, 500091 Braşov, Romania; draducanu@unitbv.ro

Received: 26 August 2020; Accepted: 21 September 2020; Published: 24 September 2020

check for updates

\begin{abstract}
In this note, we consider a subclass $\mathcal{H}_{3 / 2}(p)$ of starlike functions $f$ with $f^{\prime \prime}(0)=p$ for a prescribed $p \in[0,2]$. Usually, in the study of univalent functions, estimates on the Taylor coefficients, Fekete-Szegö functional or Hankel determinats are given. Another coefficient problem which has attracted considerable attention is to estimate the moduli of successive coefficients $\left|a_{n+1}\right|-\left|a_{n}\right|$. Recently, the related functional $\left|a_{n+1}-a_{n}\right|$ for the initial successive coefficients has been investigated for several classes of univalent functions. We continue this study and for functions $f(z)=z+\sum_{n=2}^{\infty} a_{n} z^{n} \in \mathcal{H}_{3 / 2}(p)$, we investigate upper bounds of initial coefficients and the difference of moduli of successive coefficients $\left|a_{3}-a_{2}\right|$ and $\left|a_{4}-a_{3}\right|$. Estimates of the functionals $\left|a_{2} a_{4}-a_{3}^{2}\right|$ and $\left|a_{4}-a_{2} a_{3}\right|$ are also derived. The obtained results expand the scope of the theoretical results related with the functional $\left|a_{n+1}-a_{n}\right|$ for various subclasses of univalent functions.
\end{abstract}

Keywords: univalent functions; starlike functions; coefficient estimates

MSC: 30C45; 30C50

\section{Introduction}

As usual, denote by $\mathcal{A}$ the family of all normalized analytic functions

$$
f(z)=z+\sum_{n=2}^{\infty} a_{n} z^{n}
$$

defined in the open unit disk $\mathbb{U}=\{z \in \mathbb{C}:|z|<1\}$ and let $\mathcal{S}$ be the subset of univalent functions in $\mathcal{A}$. Let

$$
\mathcal{S}^{*}(\alpha)=\left\{f \in \mathcal{A}: \Re \frac{z f^{\prime}(z)}{f(z)}>\alpha, z \in \mathbb{U}, 0 \leq \alpha<1\right\}
$$

be the class of starlike functions of order $\alpha$ (see [1]). The family $\mathcal{S}^{*}(0)=\mathcal{S}^{*}$ is the well-known class of starlike functions in $\mathbb{U}$. Denote by $\mathcal{K}$ the class of convex functions in $\mathbb{U}$, i.e.,

$$
\mathcal{K}=\left\{f \in \mathcal{A}: \Re\left(1+\frac{z f^{\prime \prime}(z)}{f^{\prime}(z)}\right)>0, z \in \mathbb{U}\right\} .
$$

In 1997, Silverman [2] investigated the properties of a subclass of $\mathcal{A}$, defined in terms of the quotient $\left(1+\frac{z f^{\prime \prime}(z)}{f^{\prime}(z)}\right) / \frac{z f^{\prime}(z)}{f(z)}$. More precisely, for $0<b \leq 1$, Silverman's class $\mathcal{G}_{b}$ is defined as follows

$$
\mathcal{G}_{b}=\left\{f \in \mathcal{A}:\left|\frac{1+\frac{z f^{\prime \prime}(z)}{f^{\prime}(z)}}{\frac{z f^{\prime}(z)}{f(z)}}-1\right|<b, z \in \mathbb{U}\right\} .
$$


In [2], Silverman proved that all functions in $\mathcal{G}_{b}$ are starlike of order $2 /(1+\sqrt{1+8 b})$. Lately, Obradović and Tuneski [3] improved the results of Silverman and obtained new starlike criteria for the class $\mathcal{G}_{b}$. Among others, they obtained the next result.

Theorem 1 ([3]). Let $f \in \mathcal{A}$. If

$$
\Re\left(\frac{1+\frac{z f^{\prime \prime}(z)}{f^{\prime}(z)}}{\frac{z f^{\prime}(z)}{f(z)}}\right)<\frac{3}{2}, z \in \mathbb{U},
$$

then $f \in \mathcal{S}^{*}$.

Starting from the above result, we consider the following subclass of $\mathcal{S}^{*}$ :

$$
\mathcal{H}_{3 / 2}=\left\{f \in \mathcal{A}: \Re\left(\frac{1+\frac{z f^{\prime \prime}(z)}{f^{\prime}(z)}}{\frac{z f^{\prime}(z)}{f(z)}}\right)<\frac{3}{2}, z \in \mathbb{U}\right\} .
$$

It is not difficult to show that for $|a|<1 / 2$, the function $f(z)=\frac{z}{1+a z} \in \mathcal{H}_{3 / 2}$.

During the years, great attention has been given to the difference of moduli of successive coefficients ||$a_{n+1}|-| a_{n}||$ of a function in $\mathcal{S}^{*}$. In 1963, Hayman [4] proved that ||$a_{n+1}|-| a_{n}|| \leq A(A \geq 1)$ for $f \in \mathcal{S}^{*}$. Further, Leung [5] proved Pommerenke's [6] conjecture ||$a_{n+1}|-| a_{n}|| \leq 1$ for $f \in \mathcal{S}^{*}$. Estimates of the difference of moduli of successive coefficients, for certain subclasses of $\mathcal{S}^{*}$, were also obtained by Z. Ye [7,8], and others (see, for example [9]). Moreover, since ||$a_{n+1}|-| a_{n}||<\left|a_{n+1}-a_{n}\right|$, the study of the functional $\left|a_{n+1}-a_{n}\right|$ has been also considered. For all functions $f \in \mathcal{K}$, Robertson [10] obtained the inequality $\left|a_{n+1}-a_{n}\right| \leq \frac{2 n+1}{3}\left|a_{2}-1\right|$ and proved that the factor $(2 n+1) / 3$ cannot be replaced by any smaller number independent of $f$. Recently, Li and Sugawa [11] investigated the problem of maximizing the functionals $\left|a_{3}-a_{2}\right|$ and $\left|a_{4}-a_{3}\right|$ for a refined subclass of $\mathcal{K}, \mathcal{K}(p)=\left\{f \in \mathcal{K}: f^{\prime \prime}(0)=p, p \in[0,2]\right\}$. The upper bounds of the same funtionals $\left|a_{3}-a_{2}\right|$ and $\left|a_{4}-a_{3}\right|$ for various subclasses of univalent functions were obtained by Peng and Obradović [12] and L. Shi et al. [13].

Motivated by the results given in [11-13], in the present paper we obtain upper bounds of the initial coefficients and upper bounds of $\left|a_{3}-a_{2}\right|$ and $\left|a_{4}-a_{3}\right|$ for a refined subclass of $\mathcal{H}_{3 / 2}$, defined by

$$
\mathcal{H}_{3 / 2}(p)=\left\{f \in \mathcal{H}_{3 / 2}: f^{\prime \prime}(0)=p\right\},
$$

where $p$ is a given number satisfying $-2 \leq p \leq 2$.

Moreover, upper bounds for functionals $\left|a_{2} a_{4}-a_{3}^{2}\right|$ and $\left|a_{4}-a_{2} a_{3}\right|$ for the same subclass $\mathcal{H}_{3 / 2}(p)$ are also derived. The first functional is known as the second Hankel determinant, studied in many papers (see [14-17]). The second functional is a particular case of the generalized Zalcman functional, investigated by Ma [18], Efraimidis and Vukotić [19] and many others (see [20-23]).

\section{Preliminary Results}

Let $\mathcal{P}$ be the class of analytic functions $p$ with a positive real part in $\mathbb{U}$, satisfying the condition $p(0)=1$. A member $p \in \mathcal{P}$ is called a Carathéodory function and has the Taylor series expansion

$$
p(z)=1+\sum_{n=1}^{\infty} p_{n} z^{n}
$$

It is known that $\left|p_{n}\right| \leq 2$ for $p \in \mathcal{P}$ and $n=1,2, \ldots$ (see [1]).

In order to prove our main results, the following two lemmas will be used. The first is due to Libera and Złotkiewicz [24,25]. 
Lemma 1. Let $-2 \leq p_{1} \leq 2$ and $p_{2}, p_{3} \in \mathbb{C}$. Then there exists a function $p \in \mathcal{P}$ of the form (7) such that

$$
2 p_{2}=p_{1}^{2}+x\left(4-p_{1}^{2}\right)
$$

and

$$
4 p_{3}=p_{1}^{3}+2\left(4-p_{1}^{2}\right) p_{1} x-\left(4-p_{1}^{2}\right) p_{1} x^{2}+2\left(4-p_{1}^{2}\right)\left(1-|x|^{2}\right) y
$$

for some $x, y \in \mathbb{C}$ with $|x| \leq 1$ and $|y| \leq 1$.

The second lemma is a special case of a more general result due to Ohno and Sugawa [26] (see also [11]).

Lemma 2. For some given real numbers $a, b, c$, let

$$
Y(a, b, c)=\max _{z \in \overline{\mathbb{U}}}\left(\left|a+b z+c z^{2}\right|+1-|z|^{2}\right)
$$

If $a c \geq 0$, then

$$
Y(a, b, c)= \begin{cases}|a|+|b|+|c|, & |b| \geq 2(1-|c|) \\ 1+|a|+\frac{b^{2}}{4(1-|c|)}, & |b|<2(1-|c|) .\end{cases}
$$

If $a c<0$, then

$$
Y(a, b, c)= \begin{cases}1-|a|+\frac{b^{2}}{4(1-|c|)}, & -4 a c\left(c^{-2}-1\right) \leq b^{2} \text { and }|b|<2(1-|c|) \\ 1+|a|+\frac{b^{2}}{4(1+|c|)}, & b^{2}<\min \left\{4(1+|c|)^{2},-4 a c\left(c^{-2}-1\right)\right\} \\ R(a, b, c), & \text { otherwise }\end{cases}
$$

where

$$
R(a, b, c)= \begin{cases}|a|+|b|-|c|, & |c|(|b|+4|a|) \leq|a b| \\ -|a|+|b|+|c|, & |a b| \leq|c|(|b|-4|a|) \\ (|c|+|a|) \sqrt{1-\frac{b^{2}}{4 a c}}, & \text { otherwise. }\end{cases}
$$

\section{Main Results}

We begin this section by finding the absolute values of the first three initial coefficients in the function class $\mathcal{H}_{3 / 2}(p)$.

Theorem 2. Let $0 \leq p \leq 2$ and let $f$, given by (1), be in the class $\mathcal{H}_{3 / 2}(p)$. Then

$$
\begin{aligned}
& \left|a_{2}\right| \leq 1 \\
& \left|a_{3}\right| \leq \frac{1}{8}\left(p^{2}+2\right) \\
& \left|a_{4}\right| \leq \frac{135 p^{3}+210 p^{2}+512}{4608}
\end{aligned}
$$

Proof. Let $f \in \mathcal{H}_{3 / 2}(p)$. Then

$$
\Re\left(\frac{1+\frac{z f^{\prime \prime}(z)}{f^{\prime}(z)}}{\frac{z f^{\prime}(z)}{f(z)}}\right)<\frac{3}{2}, z \in \mathbb{U}
$$


or equivalently

$$
\Re\left(3-2 \frac{1+\frac{z f^{\prime \prime}(z)}{f^{\prime}(z)}}{\frac{z f^{\prime}(z)}{f(z)}}\right)>0, z \in \mathbb{U} .
$$

Therefore, there exists a function $p \in \mathcal{P}$, given by (7), such that

$$
3 \frac{z f^{\prime}(z)}{f(z)}-2\left(1+\frac{z f^{\prime \prime}(z)}{f^{\prime}(z)}\right)=p(z) \frac{z f^{\prime}(z)}{f(z)} .
$$

Making use of the Taylor series representations for functions $f$ and $p$ and equating the coefficients of $z^{n}(n=1,2,3)$ on both sides of (17), we obtain

$$
\begin{gathered}
a_{2}=-\frac{p_{1}}{2} \quad a_{3}=\frac{1}{8}\left(-p_{2}-p_{1} a_{2}+6 a_{2}^{2}\right) \\
a_{4}=\frac{1}{18}\left(p_{3}+2 p_{1} a_{3}+p_{2} a_{2}-p_{1} a_{2}^{2}-30 a_{2} a_{3}+14 a_{2}^{3}\right) .
\end{gathered}
$$

Since $f \in \mathcal{H}_{3 / 2}(p)$ we have $2 a_{2}=f^{\prime \prime}(0)=p$ and then, by (18), we get $p_{1}=-2 a_{2}=-p$. In view of the last equality and Lemma 1 , we obtain

$$
\begin{gathered}
2 p_{2}=p^{2}+\left(4-p^{2}\right) x \\
4 p_{3}=-p^{3}-2\left(4-p^{2}\right) p x+\left(4-p^{2}\right) p x^{2}+2\left(4-p^{2}\right)\left(1-|x|^{2}\right) y
\end{gathered}
$$

where $x, y \in \mathbb{C}$ with $|x| \leq 1$ and $|y| \leq 1$. Making use of (18)-(21), elementary calculations yield to

$$
\begin{gathered}
a_{2}=\frac{p}{2} \quad a_{3}=\frac{1}{16}\left[3 p^{2}-\left(4-p^{2}\right) x\right] \\
a_{4}=\frac{1}{18}\left[\frac{19}{16} p^{3}-\frac{13}{16}\left(4-p^{2}\right) p x-\frac{1}{4}\left(4-p^{2}\right) p x^{2}-\frac{1}{2}\left(4-p^{2}\right)\left(1-|x|^{2}\right) y\right] .
\end{gathered}
$$

Since $p \in[0,2]$, we get $\left|a_{2}\right| \leq 1$. We have

$$
\left|a_{3}\right| \leq \frac{1}{16}\left|3 p^{2}-\left(4-p^{2}\right) x\right| \leq \frac{1}{16}\left(3 p^{2}+4-p^{2}\right)=\frac{1}{8}\left(p^{2}+2\right) .
$$

For the estimate of $\left|a_{4}\right|$, we obtain

$$
\left|a_{4}\right| \leq \frac{4-p^{2}}{36}\left(1-|x|^{2}+\left|-\frac{19 p^{3}}{8\left(4-p^{2}\right)}+\frac{13 p}{8} x+\frac{p}{2} x^{2}\right|\right) \leq \frac{4-p^{2}}{36} Y(a, b, c),
$$

where $Y(a, b, c)$ is given by $(10)$ and

$$
a=-\frac{19 p^{3}}{8\left(4-p^{2}\right)}, \quad b=\frac{13 p}{8}, c=\frac{p}{2} .
$$

Since $p \in[0,2]$, it is easy to verify that $a c<0$ and $b^{2}<\min \left\{4(1+|c|)^{2},-4 a c\left(c^{-2}-1\right)\right\}$. In view of Lemma 2, we have

$$
Y(a, b, c)=\frac{135 p^{3}+210 p^{2}+512}{128\left(4-p^{2}\right)}
$$

and thus

$$
\left|a_{4}\right| \leq \frac{4-p^{2}}{36} Y(a, b, c)=\frac{135 p^{3}+210 p^{2}+512}{4608} .
$$


Denote by

$$
\mathcal{H}_{3 / 2}(+)=\bigcup_{0 \leq p \leq 2} \mathcal{H}_{3 / 2}(p)
$$

Then, by using (15) and (16), a simple computation shows that

$$
\sup _{f \in \mathcal{H}_{3 / 2}(+)}\left|a_{3}(f)\right|=\frac{3}{4}
$$

and

$$
\sup _{f \in \mathcal{H}_{3 / 2}(+)}\left|a_{4}(f)\right|=\frac{19}{36},
$$

where $a_{3}(f)$ and $a_{4}(f)$ are the coefficients of $f$.

The upper bounds for the difference of the initial coefficients for the class $\mathcal{H}_{3 / 2}(p)$ are given in the next result.

Theorem 3. Let $0 \leq p \leq 2$ and $f(z)=z+\sum_{n=2}^{\infty} a_{n} z^{n} \in \mathcal{H}_{3 / 2}(p)$. Then,

$$
\left|a_{3}-a_{2}\right| \leq \frac{1}{4}\left(-p^{2}+2 p+1\right)
$$

and

$$
\left|a_{4}-a_{3}\right| \leq \begin{cases}\frac{-5 p^{3}+18 p^{2}-18 p+36}{144}, & p \in\left[0, \frac{2}{5}\right] \\ \frac{-135 p^{3}+606 p^{2}-612 p+1160}{4608}, & p \in\left[\frac{2}{5}, \frac{34}{21}\right] \\ \frac{-9 p^{3}+18 p^{2}+17 p-18}{72}, & p \in\left[\frac{34}{21}, 2\right] .\end{cases}
$$

Proof. Proceeding as in the proof of Theorem 2 and making use of (22), we obtain

$$
\left|a_{3}-a_{2}\right|=\frac{1}{16}\left|3 p^{2}-8 p-\left(4-p^{2}\right) x\right| \leq \frac{1}{4}\left(-p^{2}+2 p+1\right) .
$$

Now, we shall find the estimate of $\left|a_{4}-a_{3}\right|$. For this, using (22) and (23), we have

$$
\begin{gathered}
\left|a_{4}-a_{3}\right|=\frac{1}{144}\left|\frac{19 p^{3}-54 p^{2}}{2}+\frac{18-13 p}{2}\left(4-p^{2}\right) x-2\left(4-p^{2}\right) p x^{2}-4\left(4-p^{2}\right)\left(1-|x|^{2}\right) y\right| \\
\leq \frac{4-p^{2}}{36}\left[1-|x|^{2}+\left|\frac{p^{2}(54-19 p)}{8\left(4-p^{2}\right)}-\frac{18-13 p}{8} x+\frac{p}{2} x^{2}\right|\right] \leq \frac{4-p^{2}}{36} Y(a, b, c),
\end{gathered}
$$

where $Y(a, b, c)$ is given by (10) and

$$
a=\frac{p^{2}(54-19 p)}{8\left(4-p^{2}\right)}, b=-\frac{18-13 p}{8} \text { and } c=\frac{p}{2} .
$$

Since $0 \leq p \leq 2$, we have $a>0$. Note also that for $p \in[0,2]$ the inequality $|b| \geq 2(1-|c|)$ is equivalent to

$$
p \in\left[0, \frac{2}{5}\right] \cup\left[\frac{34}{21}, 2\right]
$$


Making use of Lemma 2, a computation gives

$$
Y(a, b, c)= \begin{cases}\frac{p^{2}(54-19 p)}{8\left(4-p^{2}\right)}+\frac{18-13 p}{8}+\frac{p}{2}, & p \in\left[0, \frac{2}{5}\right] \\ 1+\frac{p^{2}(54-19 p)}{8\left(4-p^{2}\right)}+\frac{(18-13 p)^{2}}{128(2-p)}, & p \in\left[\frac{2}{5}, \frac{34}{21}\right] \\ \frac{p^{2}(54-19 p)}{8\left(4-p^{2}\right)}-\frac{18-13 p}{8}+\frac{p}{2}, & p \in\left[\frac{34}{21}, 2\right] .\end{cases}
$$

Therefore, we get

$$
\left|a_{4}-a_{3}\right| \leq \begin{cases}\frac{-5 p^{3}+18 p^{2}-18 p+36}{144}, & p \in\left[0, \frac{2}{5}\right] \\ \frac{-135 p^{3}+606 p^{2}-612 p+1160}{4608}, & p \in\left[\frac{2}{5}, \frac{34}{21}\right] \\ \frac{-9 p^{3}+18 p^{2}+17 p-18}{72}, & p \in\left[\frac{34}{21}, 2\right] .\end{cases}
$$

In view of the estimates (25) and (26), we deduce that

$$
\sup _{f \in \mathcal{H}_{3 / 2}(+)}\left|a_{3}(f)-a_{2}(f)\right|=\frac{1}{2}
$$

and

$$
\sup _{f \in \mathcal{H}_{3 / 2}(+)}\left|a_{4}(f)-a_{3}(f)\right|=\frac{58 \sqrt{87}-36}{1944} \approx 0.259 \ldots
$$

where $\mathcal{H}_{3 / 2}(+)$ is given by (24) and $a_{2}(f), a_{3}(f), 4_{2}(f)$ are the coefficients of $f$.

In the next result, we obtain the estimates of the functionals $\left|a_{2} a_{4}-a_{3}^{2}\right|$ and $\left|a_{4}-a_{2} a_{3}\right|$.

Theorem 4. Let $0 \leq p \leq 2$ and let $f$, given by (1), be in the function class $\mathcal{H}_{3 / 2}(p)$. Then, the following estimates hold

$$
\begin{gathered}
\left|a_{2} a_{4}-a_{3}^{2}\right| \leq \frac{\left(6-p^{2}\right)\left(6+p^{2}\right)}{576} \\
\left|a_{4}-a_{2} a_{3}\right| \leq \begin{cases}\frac{9 p^{3}-6 p^{2}+32}{288}, & p \in\left[0, \frac{4}{3}\right] \\
\frac{p}{9}, & p \in\left[\frac{4}{3}, 2\right] .\end{cases}
\end{gathered}
$$

Proof. Proceeding again as in the proof of Theorem 2 and making use of (22) and (23), we have

$$
\begin{gathered}
\left|a_{2} a_{4}-a_{3}^{2}\right|=\frac{1}{2304}\left|-5 p^{2}+2\left(4-p^{2}\right) p^{2} x-\left(7 p^{2}+36\right)\left(4-p^{2}\right) x^{2}-32 p\left(4-p^{2}\right)\left(1-|x|^{2}\right) y\right| \\
\leq \frac{p\left(4-p^{2}\right)}{72}\left[1-|x|^{2}+\left|\frac{5 p^{3}}{32\left(4-p^{2}\right)}-\frac{p}{16} x+\frac{7 p^{2}+36}{32 p} x^{2}\right|\right]=\frac{p\left(4-p^{2}\right)}{72} Y(a, b, c),
\end{gathered}
$$


where $Y(a, b, c)$ is given by $(10)$ and

$$
a=\frac{5 p^{3}}{32\left(4-p^{2}\right)}, \quad b=-\frac{p}{16} \text { and } c=\frac{7 p^{2}+36}{32 p} .
$$

The inequality $|b| \geq 2(1-|c|)$ holds true for all $p \in[0,2]$ and therefore, from Lemma 2, we deduce that

$$
Y(a, b, c)=\frac{5 p^{3}}{32\left(4-p^{2}\right)}+\frac{p}{16}+\frac{7 p^{2}+36}{32 p}=\frac{-p^{4}+36}{8 p\left(4-p^{2}\right)}
$$

It follows that

$$
\left|a_{2} a_{4}-a_{3}^{2}\right| \leq \frac{\left(6-p^{2}\right)\left(6+p^{2}\right)}{576}
$$

To find the upper bound of $\left|a_{4}-a_{2} a_{3}\right|$ we use once more (23) and (24) and obtain

$$
\begin{gathered}
\left|a_{4}-a_{2} a_{3}\right|=\frac{1}{288}\left|-8 p^{3}-4\left(4-p^{2}\right) p x-4\left(4-p^{2}\right) p x^{2}-8\left(4-p^{2}\right)\left(1-|x|^{2}\right) y\right| \\
\leq \frac{4-p^{2}}{36}\left[1-|x|^{2}+\left|\frac{p^{3}}{4-p^{2}}+\frac{p}{2} x+\frac{p}{2} x^{2}\right|\right]=\frac{4-p^{2}}{36} Y(a, b, c),
\end{gathered}
$$

where $Y(a, b, c)$ is given by $(10)$ and

$$
a=\frac{p^{3}}{4-p^{2}} \text { and } b=c=\frac{p}{2}
$$

It easy to show that $|b|<2(1-|c|)$ for $p \in[0,4 / 3]$. An application of Lemma 2 yields

$$
Y(a, b, c)= \begin{cases}1+\frac{p^{3}}{4-p^{2}}+\frac{p^{2}}{8(2-p)}, & p \in\left[0, \frac{4}{3}\right] \\ \frac{p^{3}}{4-p^{2}}+p, & p \in\left[\frac{4}{3}, 2\right] .\end{cases}
$$

Hence, inequality (28) holds true.

Finally, using the estimates (27) and (28) we get

$$
\sup _{f \in \mathcal{H}_{3 / 2}(+)}\left|a_{2}(f) a_{4}(f)-a_{3}^{2}(f)\right|=\frac{1}{16}
$$

and

$$
\sup _{f \in \mathcal{H}_{3 / 2}(+)}\left|a_{4}(f)-a_{2}(f) a_{3}(f)\right|=\frac{2}{9},
$$

where $\mathcal{H}_{3 / 2}(+)$ is given by (24) and $a_{2}(f), a_{3}(f), 4_{2}(f)$ are the coefficients of $f$.

\section{Conclusions}

In this paper, we first considered a presumably new subclass $\mathcal{H}_{3 / 2}$ of starlike functions in the open unit disk. For a refined family $\mathcal{H}_{3 / 2}(p)(0 \leq p \leq 2)$ of $\mathcal{H}_{3 / 2}$, we investigated the upper bounds of the initial coefficients and the moduli of the initial successive coefficients. Moreover, upper bounds for functionals $\left|a_{2} a_{4}-a_{3}^{2}\right|$ and $\left|a_{4}-a_{2} a_{3}\right|$ for the same subclass $\mathcal{H}_{3 / 2}(p)$ were derived. The results obtained in this note could be a subject of further investigation related to Fekete-Szegö type functionals or Hankel determinants for the functions class $\mathcal{H}_{3 / 2}$.

Funding: This research received no external funding.

Conflicts of Interest: The author declares no conflict of interest. 


\section{References}

1. Duren, P.L. Univalent Functions; Springer: Berlin/Heidelberg, Germany; New York, NY, USA, 1983.

2. Silverman, H. Convex and starlike criteria. Int. J. Math. Math. Sci. 1999, 22, 75-79. [CrossRef]

3. Obradović, M.; Tuneski, N. On the starlike criteria defined by Silverman. Zeszyty Nauk. Politech. Rzeszowskiej Mat. 2000, 24, 59-64.

4. Hayman, W.K. On successive coefficients of univalent functions. J. Lond. Math. Soc. 1963, 38, $228-243$. [CrossRef]

5. Leung, Y. Successive coefficients of starlike functions. Bull. Lond. Math. Soc. 1978, 10, 193-196. [CrossRef]

6. Pommerenke, C. Probleme aus der Funktionen theorie. Jber. Deutsch. Math. Verein. 1971, 73, 1-5.

7. Ye, Z. On successive coefficients of close-to-convex functions. J. Math. Anal. Appl. 2003, 283, 689-695. [CrossRef]

8. Ye, Z. On successive coefficients of odd univalent functions. Proc. Am. Math. Soc. 2005, 133, 3355-3360.

9. Zaprawa, P. Successive coefficients for close-to-convex functions. Forum Math. 2020, 32, 1131-1141.

10. Robertson, M.S. Univalent functions starlike with respect to a boundary point. J. Math. Anal. Appl. 1981, 81, 327-345.

11. Li, M.; Sugawa, T. A note on successive coefficients of convex functions. Comput. Methods Funct. Theory 2017, 17, 179-193. [CrossRef]

12. Peng, Z.; Obradović, M. The estimate of the difference of initial successive coefficients of univalent functions. J. Math. Inequal. 2019, 13, 301-314. [CrossRef]

13. Shi, L.; Wang, Z.-G.; Su, R.-L.; Arif, M. Initial successive coefficients for certain classes of univalent functions involving the exponential function. arXiv 2020, arXiv:2003.09771v1.

14. Lee, S.K.; Ravichandran, V.; Supramanian, S. Bounds for the second Hankel determinant of certain univalent functions. J. Inequal. Appl. 2013, 2013, 281. [CrossRef]

15. Murugusundaramoorthy, G.; Bulboacă, T. Hankel determinants for new subclasses of analytic functions related to a shell shaped region. Mathematics 2020, 8, 1041. [CrossRef]

16. Zaprawa, P. Second Hankel determinants for the class of typically real functions. Abstr. Appl. Anal. 2016, 2016, 3792367. [CrossRef]

17. Park, J.H.; Kumar, V.; Cho, N.E. Sharp coefficient bounds for the quotient of analytic functions. Kyungpook Math. J. 2018, 58, 231-242.

18. Ma, W. Generalized Zalcman conjecture for starlike and typically real functions. J. Math. Anal. Appl. 1999, 234, 328-339. [CrossRef]

19. Efraimidis, J.; Vukotić, D. Applications of Livingstone-type inequalities to the generalized Zalcman functional. Math. Nachr. 2018, 291, 1502-1513. [CrossRef]

20. Cho, N.E.; Kwon, O.S.; Lecko, A.; Sim, Y.J. Sharp estimates of generalized Zalcman functional of early coefficients for Ma-Minda type functions. Filomat 2018, 32, 6267-6280. [CrossRef]

21. Obradović, M.; Tuneski, N. Zalcman and generalized Zalcman conjecture for the class U. arXiv 2020, arXiv:2005.14301v2.

22. Ravichandran, V.; Verma, S. Generalized Zalcman conjecture for some class of analytic functions. J. Math. Anal. Appl. 2017, 450, 592-605. [CrossRef]

23. Trąbka-Więclaw, K.; Zaprawa, P.; Gregorczyk, M.; Rysak, A. On the Fekete-Szegö type functionals for close-to-convex functions. Symmetry 2019, 11, 1497. [CrossRef]

24. Libera, R.J.; Złotkiewicz, E.J. Early coefficients of the inverse of a regular convex function. Proc. Am. Math. Soc. 1982, 85, 225-230. [CrossRef]

25. Libera, R.J.; Złotkiewicz, E.J. Coefficient bounds for the inverse of a function with derivative in P. Proc. Am. Math. Soc. 1983, 87, 251-257. [CrossRef]

26. Ohno, R.; Sugawa, T. Coefficient estimates of analytic endomorphisms of the unit disk fixing a point with applications to concave functions. Kyoto J. Math. 2018, 58, 227-241. [CrossRef]

(C) 2020 by the authors. Licensee MDPI, Basel, Switzerland. This article is an open access article distributed under the terms and conditions of the Creative Commons Attribution (CC BY) license (http:/ / creativecommons.org/licenses/by/4.0/). 